\title{
Neutrosophic Pre-open Set in Simple Extended Neutrosophic Topology
}

\author{
F. Nirmala Irudayam ${ }^{1}$, T. Madhumathi ${ }^{2}$ \\ ${ }^{1}$ Assistant Professor, Department of Mathematics, Nirmala College for Women, Coimbatore, India. \\ ${ }^{2}$ Research Scholar, Department of Mathematics, Nirmala College for Women, Coimbatore, India, \\ Email: nirmalairudayam@ymail.com ${ }^{1}$,madhumanoj1822@gmail.com²
}

\begin{abstract}
The purpose of this article is to introduce and study concept of various open sets in simple extension topology to the neutrosophic setting. This paper aims at studying the basic characterizations of these newly developed sets in the much flourishing area of recent research introduced by Smarandache. Here the relationship between these sets are also proposed. Index Terms- Neutrosophic pre ${ }^{+}$-open set, Neutrosophic pre ${ }^{+}$-closed set.
\end{abstract}

\section{INTRODUCTION}

Many real life problems in Business, Finance, Medical Sciences, Engineering and Social Sciences deal with uncertainties. There are difficulties in solving the uncertainties in these data by traditional mathematical models. To overcome these difficulties many authors have introduced many sets which deals with inconsistent data. Some of these approaches are fuzzy sets [17], Intuitionistic fuzzy sets [2], Neutrosophic sets [13] and so on which can be treated as mathematical tools to avert obstacles dealing with ambiguous data. The introduction of the idea of fuzzy set was introduced in the year 1965 by Zadeh[17]. He proposed that each element in a fuzzy set has a degree of membership. Thereafter the paper of Chang(1968)[4] paved way for the subsequent tremendous growth of the numerous fuzzy topological concepts. Following this idea K.Atanassov[1,2,3] in 1983 introduced the idea of intuitionistic fuzzy set on a universe $X$ as a generalization of fuzzy set. Here besides the degree of membership a degree of non-membership for each element is also defined. The topological framework of intuitionistic fuzzy set introduced by D. Coker[5]. The neutrosophic set was initiated by Smarandache and he explained that neutrosophic set is a generalization of intuitionistic fuzzy set. Smarandache[12,13] originally gave the definition of a neutrosophic set and neutrosophic logic. The neutrosophic logic is a formal frame trying to measure the truth, indeterminacy and falsehood. In 2012 Salama,Alblowi [14,15] introduced the concept of neutrosophic topological space. In 2016 concept of neutrosophic semi-open sets in neutrosophic topological space by introduced P.Ishwarya and K.Bageerathi[6]. In 2017 V.Venkateswara Rao and Y. Srinivasa Rao[16] introduced the concept of neutrosophic pre-open sets and neutrosophic per-closed in neutrosophic topological spaces. The concept of extending a topology by a non-open set was proposed by Levine[8] in 1963. A simple extension of a topology $\tau$ is defined as $\tau(B)=\left\{(B \cap O) \cup O^{\prime} / O, O^{\prime} \in \tau\right\}$ by Levine. F.Nirmala Irudayam [10] and Sr.I.Arockiarani introduced the concept of $b^{+}$-open sets in extended topological spaces. T. Noiri, Sr. I. Arockiarani and F. Nirmala Irudayam [11] coined the idea of $\Omega_{\mathrm{gb}}{ }^{+*}, \mho_{\mathrm{gb}}{ }^{+*}$ sets in simple extended topological spaces. B. Kanchana and F. Nirmala Irudayam [7] defined a new class of contra continuous functions via b-open sets in simple extended topological spaces. T. Madhumathi and F.Nirmala Irudayam [9] introduced the idea of $\Omega_{\mathrm{gb}}{ }^{+}$-closed sets in simple extension topological spaces. Currently, in this paper we introduced the notion of neutrosophic pre $^{+}$-open sets and neutrosophic pre $^{+}$-closed in simple extension neutrosophic topological spaces.

\section{PRELIMINARIES}

Definition 2.1 ([13]). Let $X$ be a non empty set. A neutrosophic set (NS for short) $A$ is an object having the form $A=\left\langle x, A^{1}, A^{2}, A^{3}>\right.$ where $A^{1}, A^{2}, A^{3}$ represent the degree of membership, the degree of indeterminacy and the degree of non-membership respectively of each element $\mathrm{x} \in \mathrm{X}$ of the set $\mathrm{A}$.

Definition 2.2 ([13]). Let $X$ be a non empty set, A $=\left\langle\mathrm{x}, \mathrm{A}^{1}, \mathrm{~A}^{2}, \mathrm{~A}^{3}\right\rangle$ and $\mathrm{B}=\left\langle\mathrm{x}, \mathrm{B}^{1}, \mathrm{~B}^{2}, \mathrm{~B}^{3}>\right.$ be neutrosophic sets on $X$, and let $\left\{A_{i}: i \in J\right\}$ be an arbitrary family of neutrosophic sets in $X$, where $\quad A^{i}=\left\langle x, A^{1}, A^{2}, A^{3}\right\rangle$

(i) $\mathrm{A} \subseteq \mathrm{B}$ if and only if $\mathrm{A}^{1} \leq \mathrm{B}^{1}, \mathrm{~A}^{2} \leq \mathrm{B}^{2}$ and $\mathrm{A}^{3} \geq \mathrm{B}^{3}$

(ii) $\mathrm{A}=\mathrm{B}$ if and only if $\mathrm{A} \subseteq \mathrm{B}$ and $\mathrm{B} \subseteq \mathrm{A}$.

(iii) $\bar{A}=\left\langle\mathrm{x}, \mathrm{A}^{3}, \mathrm{~A}^{2}, \mathrm{~A}^{1}\right\rangle$

(iv) $\mathrm{A} \cap \mathrm{B}=<\mathrm{x}, \mathrm{A}^{1} \wedge \mathrm{B}^{1}, \mathrm{~A}^{2} \wedge \mathrm{B}^{2}, \mathrm{~A}^{3} \vee \mathrm{B}^{3}>$

(v) $A \cup B=\left\langle x, A^{1} \vee B^{1}, A^{2} \vee B^{2}, A^{3} \wedge B^{3}>\right.$

(vi) $\cup A_{i}=\left\langle x, \vee A_{i}{ }^{1}, \vee A_{i}{ }^{2}, \wedge A_{i}{ }^{3}\right\rangle$

(vii) $\cap \mathrm{A}_{\mathrm{i}}=\left\langle\mathrm{x}, \wedge \mathrm{A}_{\mathrm{i}}{ }^{1}, \wedge \mathrm{A}_{\mathrm{i}}{ }^{2}, \vee \mathrm{A}_{\mathrm{i}}{ }^{3}\right\rangle$

(viii) $\mathrm{A} \backslash \mathrm{B}=\mathrm{A} \cap \bar{B}$.

(ix) $0_{\mathrm{N}}=\langle\mathrm{x}, 0,0,1\rangle ; 1_{\mathrm{N}}=\langle\mathrm{x}, 1,1,0\rangle$.

Definition 2.3 ([14]). A neutrosophic topology (NT for short) on a nonempty set $\mathrm{X}$ is a family $\tau$ of neutrosophic set in $\mathrm{X}$ satisfying the following axioms:

(i) $0_{\mathrm{N}}, 1_{\mathrm{N}} \in \tau$.

(ii) $G_{1} \cap G_{2} \in \tau$ for any $G_{1}, G_{2} \in \tau$.

(iii) $\cup G_{i} \in \tau$ for any arbitrary family $\left\{G_{i}: i \in J\right\} \subseteq \tau$.

In this case the pair $(\mathrm{X}, \tau)$ is called a Neutrosophic topological space (NTS for short) and any Neutrosophic 
set in $\tau$ is called a Neutrosophic open set (NOS for short) in X. The complement A of a Neutrosophic open set $A$ is called a Neutrosophic closed set (NCS for short) in X.

Definition 2.4 ([14]). Let $(X, \tau)$ be a neutrosophic topological space and $\mathrm{A}=\left\langle\mathrm{X}, \mathrm{A}_{1}, \mathrm{~A}_{2}, \mathrm{~A}_{3}\right\rangle$ be a set in $\mathrm{X}$. Then the closure and interior of $\mathrm{A}$ are defined by

$\mathrm{Ncl}(\mathrm{A})=\bigcap\{\mathrm{K}: \mathrm{K}$ is a neutrosophic closed set in $\mathrm{X}$ and $\mathrm{A} \subseteq \mathrm{K}\}$,

$\operatorname{Nint}(A)=U\{G: G$ is a neutrosophic open set in $X$ and $\mathrm{G} \subseteq \mathrm{A}\}$.

It can be also shown that $\operatorname{Ncl}(\mathrm{A})$ is a neutrosophic closed set and Nint(A) is a neutrosophic open set in $X$, and $\mathrm{A}$ is a neutrosophic closed set in $\mathrm{X}$ iff $\operatorname{Ncl}(\mathrm{A})=\mathrm{A}$; and $A$ is a neutrosophic open set in $X$ iff $\operatorname{Nint}(A)=A$.

\section{NEUTROSOPHIC PRE ${ }^{+}$-OPEN}

This section contributes to the study of the newly developed concept of neutrosophic pre ${ }^{+}$-open set of X.Futher its characterizations and its relationship with the other sets are dealt with.

Note: For any subset A of X, the interior of A is same as the interior in usual neutrosophic topology and the closure of $\mathrm{A}$ is newly defined in simple extension neutrosophic topological space(SENTS). The complement of $\mathrm{A}$ is denoted by $\mathrm{A}^{\mathrm{c}}$ or $\mathrm{X}-\mathrm{A}$ respectively.

Definition 3.1. Let A be a neutrosophic set of a simple extension neutrosophic topology. Then $\mathrm{A}$ is said to be Neutrosophic pre ${ }^{+}$open $\left[\mathrm{NP}^{+} \mathrm{O}\right]$ set of $\mathrm{X}$ if there exists a neutrosophic open set $\mathrm{NO}$ such that $\mathrm{NO} \subseteq \mathrm{A} \subseteq$ $\mathrm{NO}\left(\mathrm{Ncl}^{+}(\mathrm{A})\right)$.

Definition 3.2. A subset A of a topological space $\left(X, \tau^{+}\right)$ is said to be,

A neutrosophic pre ${ }^{+}$-open set if $\mathrm{A} \subseteq$ $\operatorname{Nint}\left(\mathrm{Ncl}^{+}(\mathrm{A})\right)$ and neutrosophic $\mathrm{pre}^{+}-$ closed set if $\mathrm{Ncl}^{+}(\operatorname{Nint}(\mathrm{A})) \subseteq \mathrm{A}$.

(ii) $\mathrm{A}$ neutrosophic $\alpha^{+}$-open set if $\mathrm{A} \subseteq$ $\operatorname{Nint}\left(\operatorname{Ncl}^{+}(\operatorname{Nint}(\mathrm{A}))\right)$ and neutrosophic $\alpha^{+}-$ closed set if $\mathrm{Ncl}^{+}\left(\operatorname{Nint}\left(\mathrm{Ncl}^{+}(\mathrm{A})\right)\right) \subseteq \mathrm{A}$.

(iii) neutrosophic semi ${ }^{+}$-open set $\mathrm{A} \subseteq$ $\mathrm{Ncl}^{+}(\operatorname{Nint}(\mathrm{A}))$ and neutrosophic semi ${ }^{+}-$ closed set if $\operatorname{Nint}\left(\mathrm{Ncl}^{+}(\mathrm{A})\right) \subseteq \mathrm{A}$.

(iv) neutrosophic $\mathrm{b}^{+}$-open set if $\mathrm{A} \subseteq$ $\mathrm{Ncl}^{+}(\operatorname{Nint}(\mathrm{A})) \cup \operatorname{Nint}\left(\mathrm{Ncl}^{+}(\mathrm{A})\right) \quad$ and neutrosophic $\mathrm{b}^{+}$-closed set $\operatorname{Ncl}^{+}(\operatorname{Nint}(\mathrm{A}))$ $\cup \operatorname{Nint}\left(\mathrm{Ncl}^{+}(\mathrm{A})\right) \subseteq \mathrm{A}$.

(v) a neutrosophic $\beta^{+}$-open set,if $A \subseteq$ $\mathrm{Ncl}^{+}\left(\operatorname{Nint}\left(\mathrm{Ncl}^{+}(\mathrm{A})\right)\right)$ and neutrosophic $\beta^{+}$closed set if $\operatorname{Nint}\left(\mathrm{Ncl}^{+}(\operatorname{Nint}(\mathrm{A}))\right) \subseteq \mathrm{A}$.

(vi) neutrosophic regular $^{+}$open set if $\mathrm{A}$ $=\operatorname{Nint}\left(\mathrm{Ncl}^{+}(\mathrm{A})\right)$ and neutrosophic regular ${ }^{+}$ closed set, if $\mathrm{A}=\mathrm{Ncl}^{+}(\mathrm{Nint}(\mathrm{A}))$.

Theorem 3.3. A subset A of a simple extension neutrosophic topological space $\mathrm{X}$ is a neutrosophic pre $^{+}$ open set if and only if $\mathrm{A} \subseteq \mathrm{Nint}\left(\mathrm{Ncl}^{+}(\mathrm{A})\right)$.

Proof: Let us consider that $\mathrm{A} \subseteq \operatorname{Nint}\left(\mathrm{Ncl}^{+}(\mathrm{A})\right)$
Now we have to prove that $\mathrm{A}$ is a neutrosophic pre ${ }^{+}$ open set in simple extension neutrosophic topological space

We know that Neutrosophic open=Nint(A). Clearly NO $\subseteq \mathrm{A} \subseteq \mathrm{NO}\left(\mathrm{Ncl}^{+}(\mathrm{A})\right)$.

Therefore $\mathrm{A}$ is $\mathrm{NP}^{+} \mathrm{O}$ set

Conversely suppose that Let $\mathrm{A}$ is a neutrosophic pre ${ }^{+}$open set in simple extension neutrosophic topological space. i.e. $\mathrm{NO} \subseteq \mathrm{A} \subseteq \mathrm{NO}\left(\mathrm{Ncl}^{+}(\mathrm{A})\right)$ for some $\mathrm{NO}$

But $\mathrm{NO} \subseteq \operatorname{Nint}(\mathrm{A})$, thus $\mathrm{NO}\left(\mathrm{Ncl}^{+}(\mathrm{A})\right) \subseteq \mathrm{Nint}\left(\mathrm{Ncl}^{+}(\mathrm{A})\right)$

Hence $\mathrm{A} \subseteq \mathrm{NO}\left(\mathrm{Ncl}^{+}(\mathrm{A})\right) \subseteq \mathrm{Nint}\left(\mathrm{Ncl}^{+}(\mathrm{A})\right)$

Therefore $A \subseteq \operatorname{Nint}\left(\mathrm{Ncl}^{+}(\mathrm{A})\right)$

Hence proved the theorem.

Theorem 3.4. In simple extension neutrosophic topology the union of two neutrosophic pre ${ }^{+}$-open sets again a neutrosophic pre ${ }^{+}$- open set.

Proof: Let A and B are neutrosophic pre ${ }^{+}$-open sets in $\mathrm{X}$ $\mathrm{A} \subseteq \operatorname{Nint}\left(\mathrm{Ncl}^{+}(\mathrm{A})\right)$

$\mathrm{B} \subseteq \operatorname{Nint}\left(\mathrm{Ncl}^{+}(\mathrm{B})\right)$

Therefore $\mathrm{A} \cup \mathrm{B} \subseteq \operatorname{Nint}\left(\mathrm{Ncl}^{+}(\mathrm{A})\right) \cup \operatorname{Nint}\left(\mathrm{Ncl}^{+}(\mathrm{B})\right)$

$\mathrm{A} \cup \mathrm{B} \subseteq \operatorname{Nint}\left(\mathrm{Ncl}^{+}(\mathrm{A}) \cup \mathrm{Ncl}^{+}(\mathrm{B})\right)$

$\mathrm{A} \cup \mathrm{B} \subseteq \operatorname{Nint}\left(\mathrm{Ncl}^{+}(\mathrm{A} \cup \mathrm{B})\right)$

Therefore by the above definition $\mathrm{A} \cup \mathrm{B}$ is also a neutrosophic pre ${ }^{+}$open set in X.

Theorem 3.5. Let $\left(\mathrm{X}, \tau^{+}\right)$be an SENTS. If $\left\{\mathrm{A}_{\alpha}\right\}_{\alpha \in \Delta}$ is a collection of a neutrosophic pre $e^{+}$-open sets in a SENTS $X$ then $\cup_{\alpha \in \Delta} A_{\alpha}$ is NPO set in X.

Proof: Let us assume that for each $\alpha \in \Delta$, we have a neutrosophic open set $\mathrm{NO}_{\alpha}$ such that

$$
\begin{aligned}
& N O_{\alpha} \subseteq A_{\alpha} \subseteq N O_{\alpha}\left(N c l^{+}(A)\right), \text { then } \\
& \cup_{\alpha \in \Delta} N O_{\alpha} \subseteq \cup_{\alpha \in \Delta} A A_{\alpha} \subseteq \cup_{\alpha \in \Delta} N O_{\alpha}\left(N c l^{+}(A)\right) \\
& \cup_{\alpha \in \Delta} A A_{\alpha} \subseteq \cup_{\alpha \in \Delta} N \operatorname{int}_{\alpha}\left(N c l^{+}(A)\right)
\end{aligned}
$$

Hence the theorem is proved.

Remark 3.6. The intersection of any two neutrosophic pre $^{+}$-open sets need not be a neutrosophic pre $^{+}$-open set in $\mathrm{X}$ as shown by the following example.

Example 3.7. Let $X=\{x\}$

$\mathrm{P}=\{\langle 0.5,0.5,0.4\rangle\}$

$\mathrm{Q}=\{\langle 0.4,0.6,0.8\rangle\}$

$\mathrm{R}=\{\langle 0.5,0.6,0.4\rangle\}$

$\mathrm{S}=\{\langle 0.4,0.5,0.8\rangle\}$

$\tau=\left\{1_{\mathrm{N}}, 0_{\mathrm{N}}, \mathrm{P}, \mathrm{Q}, \mathrm{R}, \mathrm{S}\right\}, \mathrm{B}=\{\langle 0.3,0.2,0.9\rangle\}$

$\tau^{+}=\left\{1_{N}, 0_{N}, P, Q, R, S, B\right\}$ is simple extension neutrosophic topological spaces

$\mathrm{A}_{1}=\{\langle 0.5,0.4,0.5\rangle\}$

$\left.\mathrm{A}_{2}=\{<0.5,0.3,0.4\rangle\right\}$

From this example $\mathrm{A}_{1} \cap \mathrm{A}_{2}$ is not $\mathrm{NP}^{+} \mathrm{O}$ set .

Theorem 3.8.

(i) Every neutrosophic open set in the simple extension neutrosophic topological space in $\mathrm{X}$ is neutrosophic pre ${ }^{+}$-open set in $\mathrm{X}$.

(ii) Every neutrosophic pre ${ }^{+}$-open set in the simple extension neutrosophic topological 
spaces $\left(\mathrm{X}, \tau^{+}\right)$is neutrosophic $\mathrm{b}^{+}$-open set in $\left(X, \tau^{+}\right)$.

(iii) Every neutrosophic semi ${ }^{+}$-open set in the simple extension neutrosophic topological spaces $\left(\mathrm{X}, \tau^{+}\right)$is neutrosophic $\mathrm{b}^{+}$-open set in $\left(\mathrm{X}, \tau^{+}\right)$.

(iv) Every neutrosophic $\alpha^{+}$-open set in the simple extension neutrosophic topological spaces $\left(\mathrm{X}, \tau^{+}\right)$is neutrosophic $\mathrm{b}^{+}$-open set in $\left(X, \tau^{+}\right)$.

(v) Every neutrosophic regular ${ }^{+}$-open set in the simple extension neutrosophic topological spaces $\left(\mathrm{X}, \tau^{+}\right)$is neutrosophic $\mathrm{b}^{+}$-open set in $\left(\mathrm{X}, \tau^{+}\right)$.

(vi) Every neutrosophic $\beta^{+}$-open set in the simple extension neutrosophic topological spaces $\left(\mathrm{X}, \tau^{+}\right)$is neutrosophic $\mathrm{b}^{+}$-open set in $\left(\mathrm{X}, \tau^{+}\right)$.

Proof: (i) Let A be NO set in NTS.

Then $A=\operatorname{Nint}(A)$

Clearly $\mathrm{A} \subseteq \mathrm{Ncl}^{+}(\mathrm{A})$

$\operatorname{Nint}(\mathrm{A}) \subseteq \operatorname{Nint}\left(\operatorname{Ncl}^{+}(\mathrm{A})\right)$

$\mathrm{A} \subseteq \operatorname{Nint}\left(\mathrm{Ncl}^{+}(\mathrm{A})\right)$

$\mathrm{A}$ is a neutrosophic pre ${ }^{+}$-open set in $\mathrm{X}$.

(ii) Let $\mathrm{A}$ be neutrosophic pre ${ }^{+}$-open set in a SENTS.

Then $\mathrm{A} \subseteq \operatorname{Nint}\left(\mathrm{Ncl}^{+}(\mathrm{A})\right)$ which implies

A $\subseteq \operatorname{Nint}\left(\mathrm{Ncl}^{+}(\mathrm{A})\right) \cup \operatorname{Nint}(\mathrm{A}) \subseteq \operatorname{Nint}\left(\mathrm{Ncl}^{+}(\mathrm{A})\right) \cup$ $\mathrm{Ncl}^{+}$(NintA).

Hence $\mathrm{A}$ is a neutrosophic $\mathrm{b}^{+}$-closed sets.

(iii) Let $A$ be neutrosophic semi ${ }^{+}$-open set in a SENTS.

Then $\mathrm{A} \subseteq \mathrm{Ncl}^{+}(\operatorname{Nint}(\mathrm{A}))$ which implies

A $\subseteq \operatorname{Ncl}^{+}(\operatorname{Nint}(\mathrm{A})) \cup \operatorname{Nint}(\mathrm{A}) \subseteq \operatorname{Ncl}^{+}(\operatorname{Nint}(\mathrm{A})) \cup$ $\operatorname{Nint}\left(\mathrm{Ncl}^{+} \mathrm{A}\right)$.

Hence $\mathrm{A}$ is a neutrosophic $\mathrm{b}^{+}$-closed sets.

(iv) (v) and (vi) Proof is obvious from above Definition.

Remark 3.11. From above the following implication and none of these implications is reversible as shown by examples given below

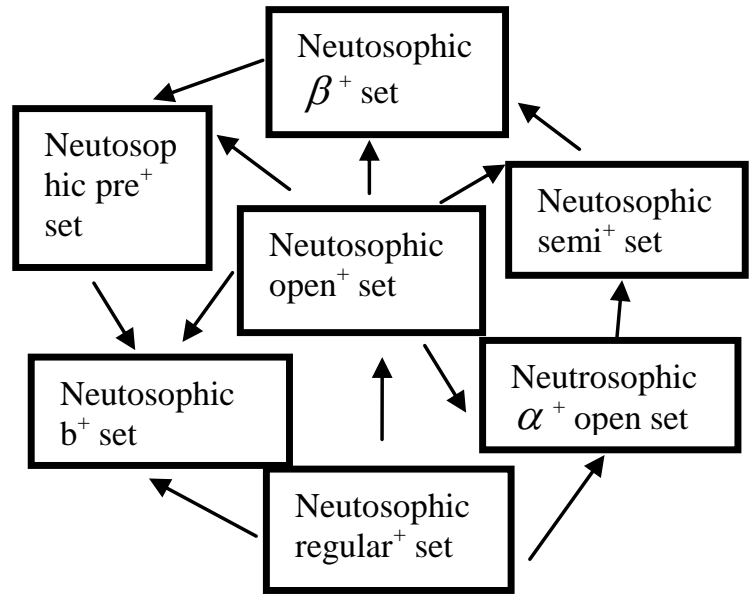

Example 3.12. Let $X=\{x\}$

$\mathrm{P}=\{\langle 0.5,0.6,0.4\rangle\}$

$\mathrm{Q}=\{\langle 0.4,0.5,0.8\rangle\}$

$\tau=\left\{1_{\mathrm{N}}, 0_{\mathrm{N}}, \mathrm{P}, \mathrm{Q}\right\}, \mathrm{B}=\{\langle 0.3,0.2,0.9\rangle\}$

$\tau^{+}=\left\{1_{N}, 0_{N}, P, Q, B\right\}$ is simple extension neutrosophic topological spaces

$\mathrm{A}=\{\langle 0.1,0.3,0.8\rangle\}$

Then the set $\mathrm{A}$ is neutrosophic ${ }^{+}$open set but not neutrosophic regular $^{+}$open set. Since $\mathrm{A}=$ $\mathrm{Ncl}^{+}(\operatorname{Nint}(\mathrm{A})) \neq 0_{\mathrm{N}}$.

Example 3.13. Let $X=\{x\}$

$\mathrm{P}=\{\langle 0.3,0.5,0.8\rangle\}$

$\mathrm{Q}=\{\langle 0.4,0.6,0.7\rangle\}$

$\tau=\left\{1_{\mathrm{N}}, 0_{\mathrm{N}}, \mathrm{P}, \mathrm{Q}\right\}, \mathrm{B}=\{\langle 0.3,0.2,0.9\rangle\}$

$\tau^{+}=\left\{1_{N}, 0_{N}, P, Q, B\right\}$ is simple extension neutrosophic topological spaces

$\mathrm{A}=\{\langle 0.1,0.3,0.5\rangle\}$

Then the set $\mathrm{A}$ is neutrosophic $\mathrm{b}^{+}$open set $\mathrm{A} \subseteq$ $\operatorname{Ncl}^{+}(\operatorname{Nint}(\mathrm{A})) \cup \operatorname{Nint}\left(\mathrm{Ncl}^{+}(\mathrm{A})\right) \subseteq 1_{\mathrm{N}}$. but not neutrosophic semi ${ }^{+}$open set. Since $A \nsubseteq \mathrm{Nintcl}^{+}\left(\mathrm{N} \mathrm{cl}^{+}\right.$ (A) $) \nsubseteq 0_{\mathrm{N}}$.

Example 3.14. Let $X=\{x\}$

$\mathrm{P}=\{\langle 0.5,0.6,0.5\rangle\}$

$\mathrm{Q}=\{\langle 0.4,0.7,0.8\rangle\}$

$\mathrm{R}=\{\langle 0.5,0.7,0.5\rangle\}$

$\mathrm{S}=\{\langle 0.4,0.6,0.8\rangle\}$

$\tau=\left\{1_{\mathrm{N}}, 0_{\mathrm{N}}, \mathrm{P}, \mathrm{Q}, \mathrm{R}, \mathrm{S}\right\}, \mathrm{B}=\{\langle 0.3,0.2,0.9\rangle\}$

$\tau^{+}=\left\{1_{N}, 0_{N}, P, Q, R, S, B\right\}$ is simple extension neutrosophic topological spaces

$\mathrm{A}=\{\langle 0.6,0.4,0.5\rangle\}$

Then the set $A$ is neutrosophic $b^{+}$open set but not neutrosophic pre ${ }^{+}$open set. Since $A \nsubseteq \mathrm{Nintcl}^{+}\left(\mathrm{N} \mathrm{cl}^{+}\right.$ (A) $) \nsubseteq<0.5,0.6,0.5\rangle$

Theorem 3.15. Let A be a neutrosophic pre ${ }^{+}$-open set in the simple extension neutrosophic topological space

$\mathrm{X}$ and suppose $A \subseteq B \subseteq N c l^{+}(A)$ then $\mathrm{B}$ is a neutrosophic pre ${ }^{+}$-open set in $\mathrm{X}$.

Proof: Let A be NO set in simple extension neutrosophic topological space $\mathrm{X}$.

Then $A=\operatorname{Nint}(A)$

Also $\mathrm{A} \subseteq \mathrm{Ncl}^{+}(\mathrm{A})$

$\operatorname{Nint}(A) \subseteq \operatorname{Nint}\left(\operatorname{Ncl}^{+}(\mathrm{A})\right)$

$\mathrm{A} \subseteq \operatorname{Nint}\left(\mathrm{Ncl}^{+}(\mathrm{A})\right)$

Hence the theorem is proved.

Lemma 3.16. Let $A$ be an NO set in $X$ and $B$ a neutrosophic pre ${ }^{+}$-open set in $\mathrm{X}$ then there exists an NO set $\mathrm{G}$ in $\mathrm{X}$ such that $B \subseteq G \subseteq \mathrm{Ncl}^{+}(B)$ it follows that

$$
A \cap B \subseteq A \cap G \subseteq A \cap N c l^{+}(B) \subseteq N c l^{+}(A \cap B)
$$

Now, since $A \cap G$ is open, from the above (theorem 3.15) lemma, $\mathrm{A} \cap \mathrm{B}$ is a neutrosophic pre $^{+}$-open set in X.

Proposition 3.17. Let $X$ and $Y$ be two simple extended neutrosophic topological spaces such that $\mathrm{X}$ is a neutrosophic product related to $\mathrm{Y}$ then the neutrosophic 
product $\mathrm{A} \times \mathrm{B}$ of a neutrosophic pre ${ }^{+}$open set $\mathrm{A}$ of $\mathrm{X}$ and a neutrosophic pre ${ }^{+}$open set $\mathrm{B}$ of $\mathrm{Y}$ is a neutrosophic pre ${ }^{+}$open set of the neutrosophic product topological space $\mathrm{X} \times \mathrm{Y}$

Proof: Let $\quad O_{1} \subseteq A \subseteq \operatorname{Ncl}^{+}\left(O_{1}\right) \quad$ and

$\mathrm{O}_{2} \subseteq B \subseteq \mathrm{Ncl}^{+}\left(\mathrm{O}_{2}\right)$

$\mathrm{O}_{1} \times \mathrm{O}_{2} \subseteq A \times B \subseteq \mathrm{Ncl}^{+}\left(\mathrm{O}_{1}\right) \times \mathrm{Ncl}^{+}\left(\mathrm{O}_{2}\right)$

$O_{1} \times O_{2} \subseteq A \times B \subseteq N c l^{+}\left(O_{1} \times O_{2}\right)$

$N \operatorname{int}\left(O_{1} \times O_{2}\right) \subseteq N \operatorname{int}(A \times B) \subseteq N \operatorname{int}\left(N c l^{+}\left(O_{1} \times O_{2}\right)\right)$

$\mathrm{O}_{1} \times \mathrm{O}_{2} \subseteq A \times B \subseteq \mathrm{Ncl}^{+}\left(\mathrm{O}_{1} \times \mathrm{O}_{2}\right)$

Hence $\mathrm{A} \times \mathrm{B}$ is neutrosophic pre ${ }^{+}$-open set in $\mathrm{X} \times \mathrm{Y}$.

Definition 3.18. Let $\left(X, \tau^{+}\right)$be a SENTS. Then for a neutrosophic subset $A$ of $X$, the neutrosophic pre $^{+}$ interior of $\mathrm{A}\left[\mathrm{NP}^{+} \mathrm{Int}(\mathrm{A})\right.$ for short ] is the union of all neutrosophic pre ${ }^{+}$-open sets of $X$ contained in $A$. That is, $\mathrm{NP}^{+}$Int $(\mathrm{A})=\bigcup\left\{\mathrm{G}: \mathrm{G}\right.$ is a $\mathrm{NP}^{+} \mathrm{O}$ set in $\mathrm{X}$ and $\mathrm{G}$ $\subseteq \mathrm{A}\}$.

Theorem 3.19. Let $\left(X, \tau^{+}\right)$be a SENTS. Then for any neutrosophic subsets $A$ and $B$ of a SENTS $X$ we have

(i) $\quad \mathrm{NP}^{+} \operatorname{int}(\mathrm{A}) \subseteq \mathrm{A}$

(ii) $\quad \mathrm{A}$ is $\mathrm{NP}^{+} \mathrm{O}$ set in $\mathrm{X} \Leftrightarrow \mathrm{NP}^{+}$int $(\mathrm{A})=\mathrm{A}$

(iii) $\quad \mathrm{NP}^{+}$int $\left(\mathrm{NP}^{+}\right.$int $\left.(\mathrm{A})\right)=\mathrm{NP}^{+}$int $(\mathrm{A})$

(iv) $\quad$ If $\mathrm{A} \subseteq \mathrm{B}$ then $\mathrm{NP}^{+}$int $(\mathrm{A}) \subseteq \mathrm{NP}^{+}$int (B)

Proof : (i) follows from Definition 3.18.

(ii)Let $\mathrm{A}^{\text {be }} \mathrm{NP}^{+} \mathrm{O}$ set in $\mathrm{X}$.

Then $\mathrm{A} \subseteq \mathrm{NP}^{+}$int $(\mathrm{A})$.

By using (i) we get $\mathrm{A}=\mathrm{NP}^{+}$int $(\mathrm{A})$.

Conversely assume that $\mathrm{A}=\mathrm{NP}^{+}$int $(\mathrm{A})$.

By using Definition 3.18. A is $\mathrm{NP}^{+} \mathrm{O}$ set in $\mathrm{X}$.

Thus (ii) is proved.

(iii)By using (ii), $\mathrm{NP}^{+}$int $\left(\mathrm{NP}^{+}\right.$int $\left.(\mathrm{A})\right)=\mathrm{NP}^{+}$int $(\mathrm{A})$. This proves (iii).

(iv) Since $\mathrm{A} \subseteq \mathrm{B}$, by using (i), $\mathrm{NP}^{+}$int (A) $\subseteq \mathrm{A} \subseteq$ B.

That is $\mathrm{NP}^{+}$int $(\mathrm{A}) \subseteq \mathrm{B}$.

By (iii), $\mathrm{NP}^{+}$int $\left(\mathrm{NP}^{+}\right.$int (A)) $\subseteq \mathrm{NP}^{+}$int (B). Thus $\mathrm{NP}^{+}$int $(\mathrm{A}) \subseteq \mathrm{NP}^{+}$int (B). This proves (iv).

Theorem 3.20. Let $\left(X, \tau^{+}\right)$be a SENTS. Then for any neutrosophic subset A and B of a SENTS, we have

(i) $\quad \mathrm{NP}^{+}$int $(\mathrm{A} \cap \mathrm{B})=\mathrm{NP}^{+}$int $(\mathrm{A}) \cap \mathrm{NP}^{+}$int (B)

(ii) $\quad \mathrm{NP}^{+}$int $(\mathrm{A} \cup \mathrm{B}) \supseteq \mathrm{NP}^{+}$int $(\mathrm{A}) \cup$ $\mathrm{NP}^{+}$int (B).

Proof: (i) Since $\mathrm{A} \cap \mathrm{B} \subseteq \mathrm{A}$ and $\mathrm{A} \cap \mathrm{B} \subseteq \mathrm{B}$,

By using Theorem 3.19 (iv), $\mathrm{NP}^{+}$int $(\mathrm{A} \cap \mathrm{B}) \subseteq$ $\mathrm{NP}^{+}$int $(\mathrm{A})$ and

$\mathrm{NP}^{+}$int $(\mathrm{A} \cap \mathrm{B}) \subseteq \mathrm{NP}^{+}$int $(\mathrm{B})$.

This implies that $\mathrm{NP}^{+}$int $(\mathrm{A} \cap \mathrm{B}) \subseteq \mathrm{NP}^{+}$int $(\mathrm{A}) \cap$ $\mathrm{NP}^{+}$int (B) -----(1).

By using Theorem 3.19 (i), $\mathrm{NP}^{+}$int $(\mathrm{A}) \subseteq \mathrm{A}$ and $\mathrm{NP}^{+}$int $(\mathrm{B}) \subseteq \mathrm{B}$.
This implies that $\mathrm{NP}^{+}$int $(\mathrm{A}) \cap \mathrm{NP}^{+}$int $(\mathrm{B}) \subseteq \mathrm{A} \cap \mathrm{B}$. Now applying Theorem 3.19 (iv), $\mathrm{NP}^{+}$int $\left(\left(\mathrm{NP}^{+}\right.\right.$int (A) $\cap \mathrm{NP}^{+}$int $\left.(\mathrm{B})\right) \subseteq \mathrm{NP}^{+}$int $(\mathrm{A} \cap \mathrm{B})$.

By (1), $\mathrm{NP}^{+}$int $\left(\mathrm{NP}^{+}\right.$int $\left.(\mathrm{A})\right) \cap \mathrm{NP}^{+}$int $\left(\mathrm{NP}^{+}\right.$int $\left.(\mathrm{B})\right) \subseteq$ $\mathrm{NP}^{+}$int $(\mathrm{A} \cap \mathrm{B})$.

By Theorem 3.19 (iii), $\mathrm{NP}^{+}$int (A) $\cap \mathrm{NP}^{+}$int (B) $\subseteq$ $\mathrm{NP}^{+}$int $(\mathrm{A} \cap \mathrm{B})$-----(2).

From (1) and (2), $\mathrm{NP}^{+}$int $(\mathrm{A} \cap \mathrm{B})=\mathrm{NP}^{+}$int $(\mathrm{A}) \cap$ $\mathrm{NP}^{+}$int (B).

This implies (i).

)(ii) Since $\mathrm{A} \subseteq \mathrm{A} \cup \mathrm{B}$ and $\mathrm{B} \subseteq \mathrm{A} \cup \mathrm{B}$,

By using Theorem 3.19 (iv), $\mathrm{NP}^{+}$int $\left(\mathrm{A} \subseteq \mathrm{NP}^{+}\right.$int ( $\mathrm{A}$ $\cup \mathrm{B})$ and

$\mathrm{NP}^{+}$int $(\mathrm{B}) \subseteq \mathrm{NP}^{+}$int $(\mathrm{A} \cup \mathrm{B})$.

This implies that $\mathrm{NP}^{+}$int $(\mathrm{A}) \cup \mathrm{NP}^{+}$int $(\mathrm{B}) \subseteq \mathrm{NP}^{+}$int $(\mathrm{A} \cup \mathrm{B})$. Hence (ii).

\section{NEUTROSOPHIC PRE ${ }^{+}$-CLOSED}

Definition 4.1. Let $A$ be neutrosophic set of a simple extension neutrosophic topology spaces $\mathrm{X}$. Then A is said to be neutrosophic pre ${ }^{+}$-closed sets of $\mathrm{X}$ if there exists a $\mathrm{NC}$ set such that $\mathrm{Ncl}^{+}(\mathrm{NC}) \subseteq \mathrm{A} \subseteq \mathrm{NC}$.

Theorem 4.2. A subset $A$ in a simple extension neutrosophic topological spaces $\mathrm{X}$ is neutrosophic pre ${ }^{+}$ closed set if and only if $\mathrm{Ncl}^{+}(\operatorname{Nint}(\mathrm{A})) \subseteq \mathrm{A}$.

Proof: Consider $\mathrm{Ncl}^{+}(\mathrm{Nint}(\mathrm{A})) \subseteq \mathrm{A}$.

Then $\mathrm{NC}=\mathrm{Ncl}^{+}(\mathrm{A})$ clearly $\mathrm{Ncl}^{+}(\mathrm{Nint}(\mathrm{A})) \subseteq \mathrm{A} \subseteq \mathrm{NC}$

Therefore $\mathrm{A}$ is $\mathrm{NP}^{+} \mathrm{C}$ set

Conversely, suppose that Let $\mathrm{A}_{\text {be }} \mathrm{NP}^{+} \mathrm{C}$ set in $\mathrm{X}$

Then $\mathrm{NC}(\mathrm{Nint}(\mathrm{A})) \subseteq \mathrm{A} \subseteq \mathrm{NC}$ for some NS closed set $\mathrm{NC}$.but $\mathrm{Ncl}^{+}(\mathrm{A}) \subseteq \mathrm{NC}$

Hence the theorem is proved.

Theorem 4.3. Let $\left(\mathrm{X}, \tau^{+}\right)$be simple extension neutrosophic topological space and $\mathrm{A}$ be a neutrosophic subset of $X$ then $A$ is a neutrosophic pre ${ }^{+}$-closed sets if and only $\mathrm{C}(\mathrm{A})$ is neutrosophic pre ${ }^{+}$-open set in $\mathrm{X}$.

Proof: Let A be a neutrosophic pre-closed set subset of $\mathrm{X}$.

Clearly $\mathrm{Ncl}^{+}(\operatorname{Nint}(\mathrm{A})) \subseteq \mathrm{A}$.

Taking complement on both sides

$\mathrm{C}(\mathrm{A}) \subseteq \mathrm{C}\left(\mathrm{Ncl}^{+}(\operatorname{Nint}(\mathrm{A}))\right)$

$\mathrm{C}(\mathrm{A}) \subseteq \operatorname{Nint}\left(\mathrm{Ncl}^{+}(\mathrm{C}(\mathrm{A}))\right)$

Hence $C(A)$ is a neutrosophic pre ${ }^{+}$-open set

Conversely suppose that $\mathrm{C}(\mathrm{A})$ is a neutrosophic pre $^{+}$open set i.e. $\mathrm{C}(\mathrm{A}) \subseteq \operatorname{Nint}\left(\mathrm{Ncl}^{+}(\mathrm{C}(\mathrm{A}))\right)$

Taking complement on both sides we get $\mathrm{Ncl}^{+}(\operatorname{Nint}(\mathrm{A}))$ $\subseteq \mathrm{A}, \mathrm{A}$ is a neutrosophic pre ${ }^{+}$-closed set

Hence the theorem is proved.

Theorem 4.4. Let $\left(\mathrm{X}, \tau^{+}\right)$be a simple extension neutrosophic topological spaces. Then intersection of two neutrosophic pre ${ }^{+}$-closed set is also a neutrosophic pre ${ }^{+}$-closed set.

Proof: Let A and B are neutrosophic pre ${ }^{+}$-closed sets on $\left(\mathrm{X}, \tau^{+}\right)$

Then $\operatorname{Ncl}^{+}(\operatorname{Nint}(\mathrm{A})) \subseteq \mathrm{A}, \mathrm{Ncl}^{+}(\operatorname{Nint}(\mathrm{B})) \subseteq \mathrm{B}$. 
Consider

$$
\begin{aligned}
& A \cap B \supseteq N c l^{+}(N \operatorname{int}(A)) \cap N c l^{+}(N \operatorname{int}(B)) \\
& \supseteq N c l^{+}(N \operatorname{int}(A) \cap N \operatorname{int}(B)) \\
& \supseteq N_{c l}(N \operatorname{int}(A \cap B))
\end{aligned}
$$

$\mathrm{Ncl}^{+}(\operatorname{Nint}(A \cap B)) \subseteq A \cap B$

Hence $A \cap B$ is a neutrosophic pre ${ }^{+}$-closed set.

Remark 4.5. The union of any two neutrosophic pre ${ }^{+}$ closed sets need not be a neutrosophic pre $^{+}$-closed set on $\left(\mathrm{X}, \tau^{+}\right)$.

Theorem 4.6. Let $\left\{\mathrm{A}_{\alpha}\right\}_{\alpha \in \Delta}$ be a collection of neutrosophic pre ${ }^{+}$-closed sets on $\left(\mathrm{X}, \tau^{+}\right)$.

then $\cap_{\alpha \in \Delta} \mathrm{A}_{\alpha}$ is a neutrosophic pre ${ }^{+}$-closed on $(\mathrm{X}$, $\left.\tau^{+}\right)$

.Proof: We have a neutrosophic set $\mathrm{NC}_{\alpha}$ such that NC ${ }_{\alpha}(\operatorname{Nint}(\mathrm{A})) \subseteq A_{\alpha} \subseteq N C_{\alpha}$ for $\quad$ all $\quad \alpha \in \Delta$ Then $\cap_{\alpha \in \Delta} N C_{\alpha}(N \operatorname{int}(A)) \subseteq \cap_{\alpha \in \Delta} A_{\alpha} \subseteq \cap_{\alpha \in \Delta} N C_{\alpha}$ $\cap_{\alpha \in \Delta} \operatorname{Ncl}_{\alpha}(N \operatorname{int}(A)) \subseteq \cap_{\alpha \in \Delta} A_{\alpha}$

Hence $\cap_{\alpha \in \Delta} A_{\alpha}$ is a neutrosophic pre ${ }^{+}$-closed sets on $\left(\mathrm{X}, \tau^{+}\right)$.

Theorem 4.7. Every neutrosophic closed set in the simple extension neutrosophic topological spaces (X, $\left.\tau^{+}\right)$is neutrosophic pre ${ }^{+}$-closed set in $\left(\mathrm{X}, \tau^{+}\right)$.

Proof: Let A be simple extension neutrosophic closed set means $A=\mathrm{Ncl}^{+}(\mathrm{A})$ and also $\operatorname{Nint}(\mathrm{A}) \subseteq \mathrm{A}$

From that, $\mathrm{Ncl}^{+}(\mathrm{Nint}(\mathrm{A})) \subseteq \mathrm{Ncl}^{+}(\mathrm{A}), \mathrm{Ncl}^{+}(\operatorname{Nint}(\mathrm{A})) \subseteq$ $\mathrm{A}$, Since $\mathrm{A}=\mathrm{Ncl}^{+}(\mathrm{A})$

Hence $A$ is a neutrosophic pre ${ }^{+}$-closed sets.

Theorem 4.8. Let $\mathrm{A}$ be a neutrosophic closed set in simple extension neutrosophic topological spaces (X, $\tau^{+}$) and suppose $\operatorname{Nint}(\mathrm{A}) \subseteq \mathrm{B} \subseteq \mathrm{A}$ then $\mathrm{B}$ is neutrosophic pre ${ }^{+}$-closed set on $\left(\mathrm{X}, \tau^{+}\right)$.

Proof: Let A be a neutrosophic set in neutrosophic topological spaces $\left(\mathrm{X}, \tau^{+}\right)$

Suppose $\operatorname{Nint}(\mathrm{A}) \subseteq \mathrm{B} \subseteq \mathrm{A}$

There exist a neutrosophic closed set NC, such that $\mathrm{NC}(\mathrm{Nint}(\mathrm{A})) \subseteq \mathrm{B} \subseteq \mathrm{A} \subseteq \mathrm{NC}$.

Then $\mathrm{B} \subseteq \mathrm{NC}$ and also $\operatorname{Nint}(\mathrm{B}) \subseteq \mathrm{B} \subseteq \mathrm{NC}$

Thus, $\mathrm{Ncl}^{+}(\mathrm{Nint}(\mathrm{B})) \subseteq \mathrm{B}$

Hence B is neutrosophic pre ${ }^{+}$-closed set on $\left(\mathrm{X}, \tau^{+}\right)$.

Theorem 4.9. Let $\mathrm{X}$ and $\mathrm{Y}$ are simple extension neutrosophic topological space such that $X$ is neutrosophic product related to $\mathrm{Y}$ then the neutrosophic product $\mathrm{A} \times \mathrm{B}$ is a neutrosophic pre ${ }^{+}$-closed set of the neutrosophic product topological space $\mathrm{X} \times \mathrm{Y}$. Where neutrosophic pre $^{+}$-closed set $\mathrm{A}$ of $\mathrm{X}$ and a neutrosophic pre $^{+}$-closed set B of $\mathrm{Y}$.

Proof: Let $\mathrm{A}$ and $\mathrm{B}$ are neutrosophic pre ${ }^{+}$-closed set. $\mathrm{C}_{1}(\mathrm{Nint}(\mathrm{A})) \subseteq \mathrm{A} \subseteq \mathrm{C}_{1}$ and $\mathrm{C}_{2}(\mathrm{Nint}(\mathrm{B})) \subseteq \mathrm{B} \subseteq \mathrm{C}_{2}$
From the above,

$\mathrm{C}_{1}(\mathrm{Nint}(\mathrm{A})) \times \mathrm{C}_{2}(\mathrm{Nint}(\mathrm{B})) \subseteq \mathrm{A} \times \mathrm{B} \subseteq \mathrm{C}_{1} \times \mathrm{C}_{2}$

$\left(\mathrm{C}_{1} \times \mathrm{C}_{2}\right)(\operatorname{Nint}(\mathrm{A} \times \mathrm{B})) \subseteq \mathrm{A} \times \mathrm{B} \subseteq \mathrm{C}_{1} \times \mathrm{C}_{2}$

Hence $\mathrm{A} \times \mathrm{B}$ is neutrosophic pre ${ }^{+}$-closed set in simple extension neutrosophic topological space $\mathrm{X} \times \mathrm{Y}$.

Definition 4.10. Let $\left(X, \tau^{+}\right)$be a SENTS. Then for a neutrosophic subset $A$ of $X$, the neutrosophic pre ${ }^{+}$closure of $\mathrm{A}\left[\mathrm{NPcl}^{+}(\mathrm{A})\right.$ for short ] is the intersection of all neutrosophic pre ${ }^{+}$-closed sets of $\mathrm{X}$ contained in $\mathrm{A}$. That is, $\mathrm{NPcl}^{+}(\mathrm{A})=\cap\left\{\mathrm{K}: \mathrm{K}\right.$ is a $\mathrm{NP}^{+} \mathrm{C}$ set in $\mathrm{X}$ and $\mathrm{K}$ $\supseteq \mathrm{A}\}$.

Theorem 4.11. Let $\left(X, \tau^{+}\right)$be a SENTS. Then for any simple extension neutrosophic subsets $\mathrm{A}$ of $\mathrm{X}$,

(i) $\quad \mathrm{C}\left(\mathrm{NP}^{+}\right.$int $\left.(\mathrm{A})\right)=\mathrm{NP}^{+} \mathrm{cl}(\mathrm{C}(\mathrm{A}))$,

(ii) $\quad \mathrm{C}\left(\mathrm{NP}^{+} \mathrm{cl}(\mathrm{A})\right)=\mathrm{NP}^{+}$int $(\mathrm{C}(\mathrm{A}))$.

Proof : By using Definition 3.18, $\mathrm{NP}^{+}$int $(\mathrm{A})=\cup\{\mathrm{G}$ : $\mathrm{G}$ is a $\mathrm{NP}^{+} \mathrm{O}$ set in $\mathrm{X}$ and $\left.\mathrm{G} \subseteq \mathrm{A}\right\}$.

Taking complement on both sides,

$\mathrm{C}\left(\mathrm{NP}^{+}\right.$int $\left.(\mathrm{A})\right)=\mathrm{C}\left(\cup\left\{\mathrm{G}: \mathrm{G}\right.\right.$ is a $\mathrm{NP}^{+} \mathrm{O}$ set in $\mathrm{X}$ and $\mathrm{G}$ $\subseteq \mathrm{A}\})$

$\mathrm{C}\left(\mathrm{NP}^{+}\right.$int $\left.(\mathrm{A})\right)=\cap\left\{\mathrm{C}(\mathrm{G}): \mathrm{C}(\mathrm{G})\right.$ is a $\mathrm{NP}^{+} \mathrm{C}$ set in $\mathrm{X}$ and $\mathrm{C}(\mathrm{A}) \subseteq \mathrm{C}(\mathrm{G})\}$.

Replacing $\mathrm{C}(\mathrm{G})$ by $\mathrm{K}$, we get $\mathrm{C}\left(\mathrm{NP}^{+}\right.$int $\left.(\mathrm{A})\right)=\cap\{\mathrm{K}$ : $\mathrm{K}$ is a $\mathrm{NP}^{+} \mathrm{C}$ set in $\mathrm{X}$ and $\left.\mathrm{K} \supseteq \mathrm{C}(\mathrm{A})\right\}$.

By Definition 4.10, $\mathrm{C}\left(\mathrm{NP}^{+}\right.$int $\left.(\mathrm{A})\right)=\mathrm{NP}^{+} \mathrm{cl}(\mathrm{C}(\mathrm{A}))$.

This proves (i). By using (i), $\mathrm{C}\left(\mathrm{NP}^{+}\right.$int $\left.(\mathrm{C}(\mathrm{A}))\right)=\mathrm{NP}^{+} \mathrm{cl}$ $(\mathrm{C}(\mathrm{C}(\mathrm{A})))=\mathrm{NP}^{+} \mathrm{cl}(\mathrm{A})$.

Taking complement on both sides, we get $\mathrm{NP}^{+}$int $(\mathrm{C}(\mathrm{A}))=\mathrm{C}\left(\mathrm{NP}^{+} \mathrm{cl}(\mathrm{A})\right)$. Hence proved (ii).

Theorem 4.12. Let $\left(X, \tau^{+}\right)$be a SENTS. Then for any simple extension neutrosophic subsets $\mathrm{A}$ and $\mathrm{B}$ of a SENTS $X$ we have
(i) $\quad \mathrm{A} \subseteq \mathrm{NP}^{+} \mathrm{cl}(\mathrm{A})$
(ii) $\quad \mathrm{A}$ is $\mathrm{NP}^{+} \mathrm{C}$ set in $\mathrm{X} \Leftrightarrow \mathrm{NP}^{+} \mathrm{cl}(\mathrm{A})=\mathrm{A}$
(iii) $\quad \mathrm{NP}^{+} \mathrm{cl}\left(\mathrm{NP}^{+} \mathrm{cl}(\mathrm{A})\right)=\mathrm{NP}^{+} \mathrm{cl}(\mathrm{A})$
(iv) $\quad$ If $\mathrm{A} \subseteq \mathrm{B}$ then $\mathrm{NP}^{+} \mathrm{cl}(\mathrm{A}) \subseteq \mathrm{NP}^{+} \mathrm{cl}(\mathrm{B})$

Proof : (i) follows from Definition 4.10.

(ii)Let $\mathrm{A}$ be $\mathrm{NP}^{+} \mathrm{C}$ set in $\mathrm{X}$.

By using Theorem 4.3, C(A) is $\mathrm{NP}^{+} \mathrm{O}$ set in $\mathrm{X}$.

By Theorem 4.11 (ii), $\mathrm{NP}^{+}$int $(\mathrm{C}(\mathrm{A}))=\mathrm{C}(\mathrm{A}) \Leftrightarrow$ $\mathrm{C}\left(\mathrm{NP}^{+} \mathrm{cl}(\mathrm{A})\right)=\mathrm{C}(\mathrm{A}) \Leftrightarrow \mathrm{NP}^{+} \mathrm{cl}(\mathrm{A})=\mathrm{A}$.

Thus proved (ii).

(iii)By using (ii), $\mathrm{NP}^{+} \mathrm{cl}\left(\mathrm{NP}^{+} \mathrm{cl}(\mathrm{A})\right)=\mathrm{NP}^{+} \mathrm{cl}$ (A). This proves (iii).

(iv)Since $\mathrm{A} \subseteq \mathrm{B}, \mathrm{C}(\mathrm{B}) \subseteq \mathrm{C}(\mathrm{A})$.

By using Theorem 3.19 (iv), $\mathrm{NP}^{+}$int $(\mathrm{C}(\mathrm{B})) \subseteq \mathrm{NP}^{+}$int (C(A)).

Taking complement on both sides, $\mathrm{C}\left(\mathrm{NP}^{+}\right.$int $\left.(\mathrm{C}(\mathrm{B}))\right) \supseteq$ $\mathrm{C}\left(\mathrm{NP}^{+}\right.$int $\left.(\mathrm{C}(\mathrm{A}))\right)$.

By Theorem 4.11 (ii), $\mathrm{NP}^{+} \mathrm{cl}(\mathrm{A}) \subseteq \mathrm{NP}^{+} \mathrm{cl}$ (B). This proves (iv).

Theorem 4.13. Let $\left(X, \tau^{+}\right)$be a SENTS. Then for a simple extension neutrosophic subset $\mathrm{A}$ and $\mathrm{B}$ of a SENTS X, we have

(i) $\quad \mathrm{NP}^{+} \mathrm{cl}(\mathrm{A} \cup \mathrm{B})=\mathrm{NP}^{+} \mathrm{cl}(\mathrm{A}) \cup \mathrm{NP}^{+} \mathrm{cl}(\mathrm{B})$ and 
(ii) $\quad \mathrm{NP}^{+} \operatorname{cl}(\mathrm{A} \cap \mathrm{B}) \subseteq \mathrm{NP}^{+} \operatorname{cl}(\mathrm{A}) \cap \mathrm{NP}^{+} \mathrm{cl}$ (B).

Proof : Since $\mathrm{NP}^{+} \mathrm{cl}(\mathrm{A} \cup \mathrm{B})=\mathrm{NP}^{+} \mathrm{cl}(\mathrm{C}(\mathrm{C}(\mathrm{A} \cup$ B))),

By using Theorem 4.11 (i), $\mathrm{NP}^{+} \mathrm{cl}(\mathrm{A} \cup \mathrm{B})=$ $\mathrm{C}\left(\mathrm{NP}^{+}\right.$int $\left.(\mathrm{C}(\mathrm{A} \cup \mathrm{B}))\right)$

$\mathrm{NP}^{+} \mathrm{cl}(\mathrm{A} \cup \mathrm{B})=\mathrm{C}\left(\mathrm{NP}^{+}{ }^{+}\right.$int $\left.(\mathrm{C}(\mathrm{A}) \cup \mathrm{C}(\mathrm{B}))\right)$.

Again using Theorem 3.20 (i), $\mathrm{NP}^{+} \mathrm{cl}(\mathrm{A} \cup \mathrm{B})=$ $\mathrm{C}\left(\mathrm{NP}^{+}\right.$int $(\mathrm{C}(\mathrm{A})) \cap \mathrm{NP}^{+}$int $\left.(\mathrm{C}(\mathrm{B}))\right)$

$\mathrm{NP}^{+} \mathrm{cl}(\mathrm{A} \cup \mathrm{B})=\mathrm{C}\left(\mathrm{NP}^{+} \operatorname{int}(\mathrm{C}(\mathrm{A}))\right) \cup \mathrm{C}\left(\mathrm{NP}^{+}\right.$int $(\mathrm{C}$ (B))).

By using Theorem 4.11 (i), $\mathrm{NP}^{+} \mathrm{cl}(\mathrm{A} \cup \mathrm{B})=$ $\mathrm{NP}^{+} \mathrm{cl}(\mathrm{C}(\mathrm{C}(\mathrm{A}))) \cup \mathrm{NP}^{+} \mathrm{cl}(\mathrm{C}(\mathrm{C}(\mathrm{B})))$

$\mathrm{NP}^{+} \mathrm{cl}(\mathrm{A} \cup \mathrm{B})=\mathrm{NP}^{+} \mathrm{cl}$ (A) $\cup \mathrm{NP}^{+} \mathrm{cl}$ (B). Thus proved (i).

(ii) Since $\mathrm{A} \cap \mathrm{B} \subseteq \mathrm{A}$ and $\mathrm{A} \cap \mathrm{B} \subseteq \mathrm{B}$,

By using Theorem 4.12 (iv), $\mathrm{NS} \mathrm{Cl}(\mathrm{A} \cap \mathrm{B}) \subseteq \mathrm{NP}^{+} \mathrm{cl}$ (A) and $\mathrm{NP}^{+} \mathrm{cl}(\mathrm{A} \cap \mathrm{B}) \subseteq \mathrm{NP}^{+} \mathrm{cl}(\mathrm{B})$.

This implies that $\mathrm{NP}^{+} \mathrm{cl}(\mathrm{A} \cap \mathrm{B}) \subseteq \mathrm{NP}^{+} \mathrm{cl}(\mathrm{A}) \cap$ $\mathrm{NP}^{+} \mathrm{cl}$ (B). This proves(ii).

\section{CONCLUSION}

With the induction of the above definitions in simple extension neutrosophic topological spaces. We can extend its scope by generalizing this new concept. This would open new avenues of research in the existing neutrosophic topological setting.

\section{REFERENCES}

[1] K. Atanassov, Intuitionistic fuzzy sets, in V.Sgurev, ed., VII ITKRS session, sofia (June 1983 central Sci. and Tech. Library, Bulg, Academy of sciences (1984)).

[2] K. Atanassov, Intuitionistic fuzzy sets, Fuzzy sets and systems 20(1986) 81-86.

[3] K.Atanassov, Review and new result on intuitionistic fuzzy sets, preprint Im-MFAIS-188, Sofia, 1988.

[4] C.L. Chang, Fuzzy Topological Spaces, J. Math. Anal Appl.,24 (1968), 182-1 90.

[5] Dogan Coker, An introduction to Intuitionistic fuzzy topological spaces, Fuzzy sets and systems, 88, 81-89 (1997).

[6] P.Ishwarya and K.Bageerathi, On Neutrosophic semi-open sets in Neutrosophic topological spaces, International Journal of Mathematics Trends and Technology, Vol 37(3), 214223(2016).

[7] B. Kanchana and F. Nirmala Irudayam, “A new class of contra continuous Functions via b-open sets in SETS", International Journal of Science and Research, Vol.5, Issue.8, Aug 2016, 12401245.

[8] N. Levine , "Simple extension of topology", Amer.math.Monthly, 71 (1964),22-105.

[9] T. Madhumathi and F.Nirmala Irudayam, “On $\Omega_{\mathrm{gb}}{ }^{+}$-closed sets in simple extension topologicalspaces,'ElixirAppl.Math.105(2017) ,46305-46307.
[10] F. Nirmala Irudayam and Sr.I Arokiarani, "A note on the weaker form of bI set and its generalization in SEITS", International Journal of Computer application, Issue 2, vol.4(Aug 2012),42-54.

[11] Noiri T., Arockiarani Sr.I., and Nirmala Irudayam F., "On Some Topologies Induced

By $\mathrm{BI}^{+}$Open Sets in Simple Extension Ideal Topological Spaces", International Journal of Mathematical Archive-4(1), 2013, 30-135.

[12] Florentin Smarandache, neutrosophy and neutrosophic Logic, First International conference on neutrosophy, Neutrosophic Logic, set, probability and statistics university of New mexico, Gallup NM 87301, USA(2002).

[13] F. Smarandache. A Unifying Field in Logics: Neutrosophic Logic. Neutrosophy, Neutrosophic Set, Neutrosophic Probability, American Research Press, Rehoboth, NM (1999).

[14] A.A. Salama, S.A. AL-Blowi, Neutrosophic Set and Neutrosophic Topological Spaces, IOSR Journal of Math., 3 (2012), 31-35.

[15] A. A. Salama, S. A. Alblowi, Generalized Neutrosophic Set and Generalized Neutrosophic Topological Spaces, Comp. Sci. Engg., 2 (2012), 129-132.

[16] V. Venkateswara Rao and Y. Srinivasa Rao, Neutrosophic Pre-open Sets and Pre-closed Sets in Neutrosophic Topology, International Journal of ChemTech Research, Vol.10 No.10, pp: 449-458, 2017.

[17] L.A. Zadeh, Fuzzy sets, Information and control 8(1965) 338-353. 